\title{
Subcelluar localization of orf126 of Bombyx mori nucleopolyhedrovirus
}

\author{
HAO Bifang ${ }^{1 \star}$, GUO XiaoFang ${ }^{2}$, WANG Meng ${ }^{1}$ and SHEN Xingjia ${ }^{1}$ \\ ${ }^{1}$ Sericultural Research Institute, Jiangsu University of Science and Technology, Zhenjiang Jiangsu 212018, China. \\ ${ }^{2}$ School of Computer Science and Engineering, Jiangsu University of Science and Technology, Zhenjiang Jiangsu \\ 212003, China. \\ Accepted 1 July, 2011

\begin{abstract}
In order to explore the mechanism of orf126 of Bombyx mori nucleopolyhedro virus, the subcellular localization of ORF126 was conducted. The egfp gene was fused with the C-terminal of orf126 genes, $\mathrm{BmN}$ cells were transfected with different plasmid DNA and the superinfection were performed at $12 \mathrm{~h}$ post transfection. The fluorescence was examined by confocal laser scanning microscopy at different time point after transfection. The results show that EGFP protein was uniformly present throughout the cytoplasm and nucleus either in expression alone or superinfection, however, the fluorescence of EGFP linked to ORF126s were present barely in the cytoplasm.
\end{abstract}

Key words: BmNPV, orf126, transient expression, subcellular localization.

\section{INTRODUCTION}

Baculoviruses are double-stranded DNA viruses that are pathogenic for invertebrates, particularly insects of the order Lepidoptera. Bombyx mori nucleopolyhedrovirus (BmNPV), a member of baculovirus, is an important pathogen of silkworm, which results in about 5 to $15 \%$ lost in the sericulturer in China. A number of genes' function have been depicted since its genomics sequence was reported (Gomi et al., 1999); these results rebound to keep abreast the virus life cycle and to prevent the occurrence of the disease. Our previous research revealed that BmNPV orf126 was not a necessary gene for virus replication (Hao et al., 2009) and two subtypes (SX126 and GD126) were found in seven wild isolates collected from different provinces of China, which exhibited different function in the virus replication cycle; GD126 can enhance the polyhedrin expression significantly, but its mechanism still remain unclear, thus further investigation is required for exploring the mechanism of orf126. Baculovirus gene expression is regulated mainly at the transcriptional level, and involves sequential expression of early, late and very late genes. The function of early genes is the initiation of infection, and they are required to accelerate replication events and to prepare

\footnotetext{
*Corresponding author. E-mail: bifanghao@gmail.com
}

the host cell for virus multiplication (Friesen, 1997). Orf126 is an early gene, whose product was detected from $6 \mathrm{~h}$ post infection (Hao et al., 2009), thus Orf126 was supposed to be involved in the replication of virus or interacted with host cell. Comprehensive knowledge of the location of proteins within the cellular microenvironments is critical for understanding their functions, and the location will provide the necessary information for the interaction of protein and the evolution. However, the location of ORF126 exerting its function which is required for the functional assay of ORF126 in the host cell is still unclear.

Together with various experimental approaches to determine the subcellular localization of proteins, many powerful computational tools have been developed for predicting the subcellular localization of proteins in various organisms based on the sequence information alone (Chou and Shen, 2008; Chou and Elrod 1999; Chou et al., 2011; Chou, 2001; Jiang et al., 2008; Li et al., 2008; Zeng et al., 2009) as well as a long list of references cited in a comprehensive review (Chou and Shen, 2007). Among the aforementioned predictors, the predictor iLoc-Euk (Chou et al., 2011), some predictors in the Cell-PLoc package (Chou and Shen, 2008) and all the predictors in the Cell-PLoc 2.0 package (Chou and Shen, 2010) can be used for not only the single-location or "singleplex" proteins but also the multiple-location or 


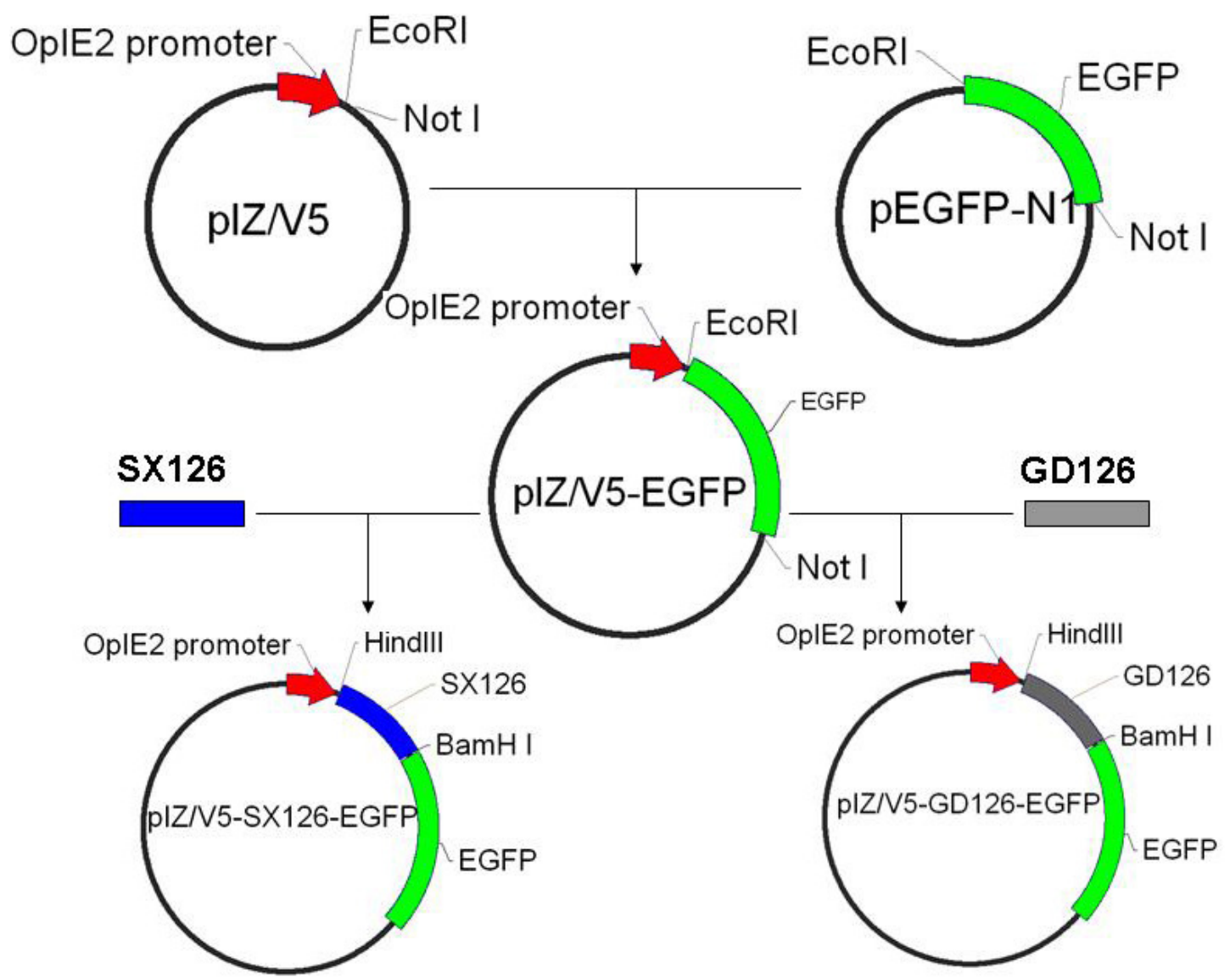

Figure 1. Procedure of the construction of transfection vector.

"multiplex" proteins that may simultaneously reside at, or move between two or more different subcellular locations. Proteins with multiple location sites or dynamic feature of this kind are particularly interesting because they may have some unique biological functions (Glory and Murphy., 2007 ). Particularly, as pointed out by Millar et al. (2009), recent evidences have indicated that an increasing number of proteins have multiple locations in the cell. EGFP was an efficient molecular probe; it was applied widely in the localization analysis through its fusion with other proteins (Cheng et al., 2009; Huang et al., 2008; Petry et al., 2006). In this study, the localization of ORF126 was explored by the EGFP probe, the location was examined through the scanning of EGFP in the $\mathrm{BmN}$ cell by laser confocal microscope, and the results provided the subcelluar location of ORF126 where it exerted its function.

\section{MATERIALS AND METHODS}

\section{Insect cell line and virus}

$\mathrm{BmN}$ cells were maintained at $28^{\circ} \mathrm{C}$ in TC-100 insect medium (PAA Company) supplemented with $10 \%$ fetal bovine serum (FBS; Gibco$\mathrm{BRL}$ ). The wild-type (wt) BmNPV strains used in the experiments were originally isolated from the cadavers of $B$. mori larvae collected in Guangdong, Shaanxi provinces of China and were designated BmNPV-GD and BmNPV-SX, respectively, and vBmBacJS13-ph was constructed by Huang et al. (2007).

\section{Construction of the transient expression vector}

The plasmids containing the different subtypes of orf126-egfp fusion gene were constructed to study the localization of ORF126 in BmN cells (Figure 1). A fragment containing the egfp gene was digested from pEGFP-N1 (Clontech) with EcoRI and Notl, and subcloned into plZ/V5-His (Invitrogen) to generate pIZ/V5-egfp. The SX126 or GD126 sequence without the stop codon (TAA) was amplified by PCR from viral DNAs which were extracted from purified OBs of isolates BmNPV-GD and BmNPV-SX as described by Sun and Zhang (1994) using the primer pair 126SF (5'-GCGAAGCTTA TGTTCAAACCCAACAT GATTAGA-3'; HindIII site underlined) and 126SR (5'-GCGGGATCCGCATAAATTTTATTTTTATTTTTGTTTT GGTT-3'; BamHI site underlined). The PCR products were purified and cloned into the pIZ/V5-egfp to generate pIZ/V5-SX126-EGFP or pIZ/V5-GD126-EGFP.

\section{Localization analysis of ORF126}

Plasmid plZ/V5-SX126-EGFP or pIZ/V5-GD126-EGFP was transfected into $\mathrm{BmN}$ cells with lipofectin (Invitrogen) following the manufacturer's protocol. Plasmid plZ/V5-egfp was used as a 


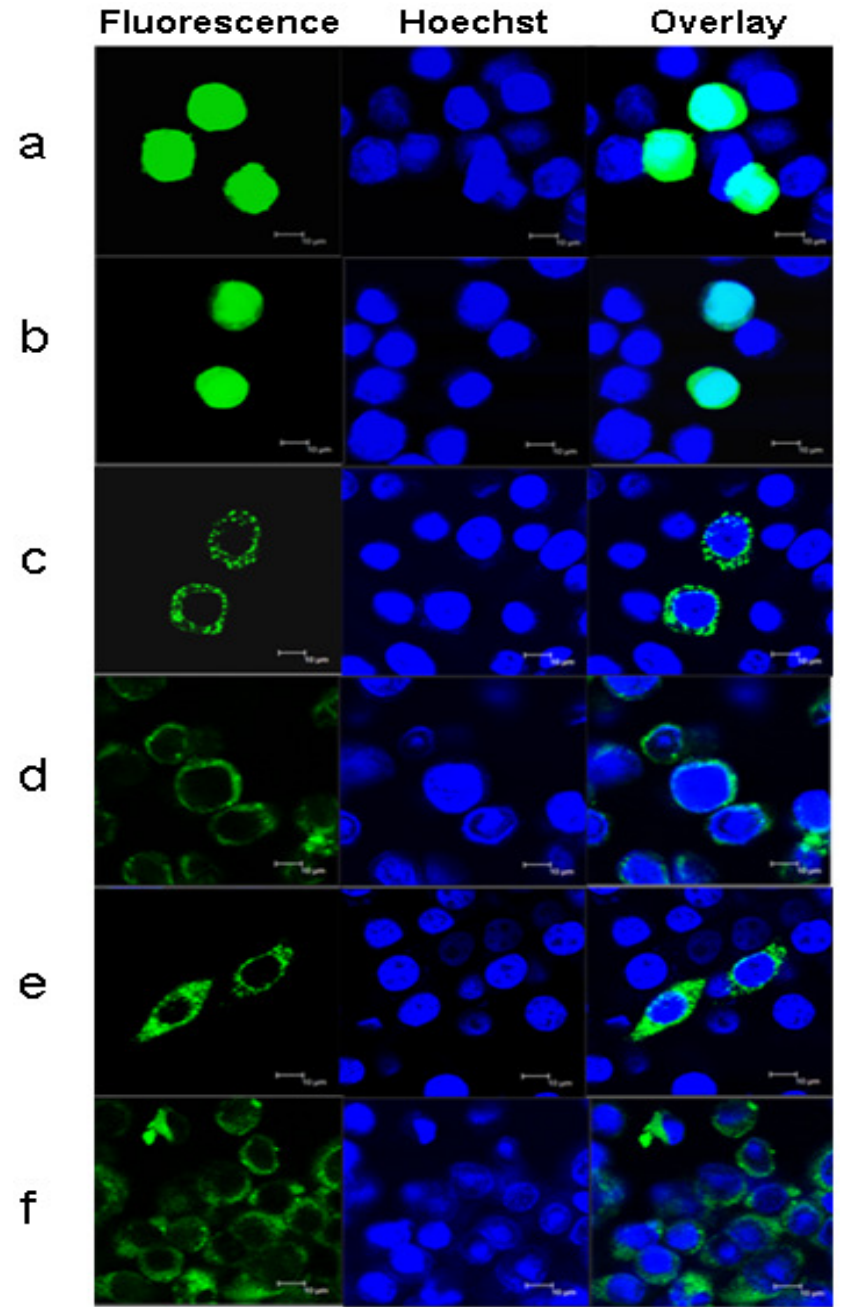

Figure 2. Localization of the SX126 and GD126 EGFP-fusion proteins in $\mathrm{BmN}$ cells with or without virus infection. BmN cells were transfected with the control plasmid plZ/V5-egfp (a and b), the plasmid pIZ/V5-SX126-EGFP (c and d) or with pIZ/V5GD126-EGFP (e and f). BmN cells (b, $d$ and f) were infected with BmBacJS13-ph at $12 \mathrm{~h}$ p.t., the nuclear DNAs of the cells were stained with Hoechst and were examined by confocal laser scanning microscopy for fluorescence at $60 \mathrm{~h}$ p.t. All magnification and exposure times were identical and bars $=10$ $\mu \mathrm{m}$.

negative control. Superinfection was conducted with vBmBacJS13ph (MOI of 10 TCID $_{50}$ units per cell) at $12 \mathrm{~h}$ post-transfection (p.t.). The sample was observed at a time interval of $12 \mathrm{~h}$. The cell samples for confocal examination were fixed by paraformaldehyde of $4 \%$ for $15 \mathrm{~min}$, rinsed with PBS, stained with Hoechst (Beyotime) dye for 5 min, washed with PBS for two times and observed under a confocal laser-scanning microscope (Leica).

\section{RESULTS AND DISCUSSION}

The transient expression vector was identified by double digestion of restriction endonuclease analysis (data not shown), then the correct plasmids were applied to transfect $\mathrm{BmN}$ cell, and superinfection was conducted at $12 \mathrm{~h}$ p.t. The fluorescence was examined by confocal laser-scanning microscopy at $60 \mathrm{~h}$ p.t. The results show that both the intracellular localization of SX126 and GD126 ORF126 were localized in the cytoplasm of transfected cells through transient expression, and fluorescence congregation was also observed in the cytoplasm. As shown in Figure 2, the fusion proteins of SX126 or GD126 were located exclusively in the cytoplasm of transfected cells (Figure $2 c$ and e). When $\mathrm{BmN}$ cells were superinfected with vBmBacJS13-ph, fluorescence remained in the cytoplasm of infected cells (Figure $2 \mathrm{~d}$ and f), hereby, their location was not affected by other factors of the virus. However, in the negative controls, EGFP showed homogeneous fluorescence in the cytoplasm and nucleus when expressed alone (Figure 2a) or when superinfected with vBmBacJS13-ph (Figure 2b). Therefore, ORF126 is likely to be localized in the cytoplasm of the host cells. The bioinformatics analysis showed that ORF126 has a endoplasmic reticulum location signal (KNKNKIY) in the C-terminal, which was not in the Ac150, however, the truncated ORF126 localization was not changed (data not shown), so ORF126 might contain other cytoplasm location sequence, which will be distinguished by further investigation. Though there are small different amino acid difference between SX126 and GD126, their phenotype is completely different, but the similar subcellular location indicated that they were not involved in the virus replication cycle since they did not enter the nuclear. Combined with the cytopathic effect of the cell infected with GD126-contained virus (cell lysed time or the $\mathrm{ST}_{50}$ of the infected larvae), ORF126 may interact with the host cell factor in the cytoplasm, but further investigation is needed.

\section{REFERENCES}

Cheng F, Mclaughlin PJ, Verderame MF, Zagon IS (2009). Dependence on nuclear localization signals of the opioid growth factor receptor in the regulation of cell prolification. Exp. Biol. Med. 234(5): 532-541.

Chou KC, Wu ZC, Xiao X (2011). iLoc-Euk: A Multi-Label Classifier for Predicting the Subcellular Localization Singleplex Multiplex Eukaryotic Proteins. PLoS One. 6: e18258.

Chou KC (2001). Prediction of protein cellular attributes using pseudo amino acid composition. Proteins: Structure, Function, Gen. (43): 246-255.

Chou KC, Shen HB (2010). Cell-PLoc 2.0: An improved package of web-servers for predicting subcellular localization of proteins in various organisms. Natural Sci (2): 1090-1103.

Chou KC, Elrod DW (1999). Protein subcellular location prediction. Protein. Eng. (12): 107-118.

Chou KC, Shen HB (2007). Review Recent progresses in protein subcellular location prediction. Anal. Biochem. (370): 1-16.

Chou KC, Shen HB (2008). Cell-PLoc: A package of Web servers for predicting subcellular localization of proteins in various organisms. Nature Protocols, (3): 153-162.

Friesen PD (1997). Regulation of Baculovirus Early Gene Expression. New York: Plenum.

Glory E, Murphy RF (2007). Automated subcellular location determination and high-throughput microscopy. Dev Cell. (12): 7-16.

Gomi S, Majima K, Maeda S (1999). Sequence analysis of the genome 
of Bombyx mori nucleopolyhedrovirus. J. Gen. Virol. 80(5): 13231337.

Hao B, Huang J, Sun X, Deng F, Zhang Y, Wang H, Chen $\mathrm{H}, \mathrm{Hu} Z$ (2009). Variants of open reading frame $\mathrm{Bm} 126$ in wild-type BmNPV isolates exhibit functional differences. J. Gen. Virol. 90(1): 153-161.

Huang J, Hao B, Deng F, Sun X, Wang H, Hu Z (2008). Open reading frame Bm2 1 of Bombyx mori nucleopolyhedrovirus is not essential for virus replication in vitro, but its deletion extends the median survival time of infected larvae. J. Gen. Virol. 89(4): 922-930.

Huang J, Hao B, Sun X, Deng F, Wang H and Hu Z (2007). Construction of the Bac-to-Bac system of Bombyx mori nucleopolyhedrovirus. Virol. Sin. 22(3): 218-225.

Jiang X, Wei R, Zhang TL, Gu Q (2008). Using the concept of Chou's pseudo amino acid composition to predict apoptosis proteins subcellular location: an approach by approximate entropy. Protein \& Peptide Letters. (15): 392-396.

Li MQZ (2008). Predicting protein subcellular location using Chou's pseudo amino acid composition and improved hybrid approach. Protein \& Peptide Letters. (15): 612-616.
Millar AH, Carrie C, Pogson B, Whelan J (2009). Exploring the functionlocation nexus: using multiple lines of evidence in defining the subcellular location of plant proteins. Plant Cell. (21): 1625-1631.

Petry F, Ritz V, Meineke C, Middel P, Kietzmann T, Schmitz-Salue C, Hirsch-Ernst KI (2006). Subcellular localization of rat Abca5, a rat ATP-binding-cassette transporter expressed in Leydig cells, and characterization of its splice variant apparently encoding a halftransporter. Biochem. J. (393): 79-87.

Sun X, Zhang G (1994). Comparison of four wild isolates of Heliothis nuclear polyhedrosis virus. Virol. Sin. 9: 309-318.

Zeng YH, Guo YZ, Xiao RQ, Yang L, Yu LZ, Li ML (2009). Using the augmented Chou's pseudo amino acid composition for predicting protein submitochondria locations based on auto covariance approach. J. Theor. Biol. (259): 366-372. 